\title{
Levels of plasma Epstein-Barr virus DNA prior and subsequent to treatment predicts the prognosis of nasopharyngeal carcinoma
}

\author{
FEI-PENG ZHAO ${ }^{1,2^{*}}$, XIONG LIU $^{1 *}$, XIAO-MEI CHEN $^{1 *}$, JUAN LU $^{1}$, BO-LONG YU ${ }^{1}$, WEN-DONG TIAN ${ }^{1}$,

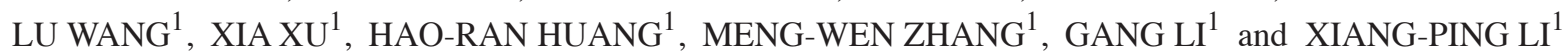 \\ ${ }^{1}$ Department of Otolaryngology - Head and Neck Surgery, Nanfang Hospital, Southern Medical University, \\ Guangzhou, Guangdong 510515; ${ }^{2}$ Department of Otolaryngology - Head and Neck Surgery, \\ The Affiliated Hospital of Luzhou Medical College, Luzhou Medical College, Luzhou, Sichuan, 646000, P.R. China
}

Received July 19, 2014; Accepted March 26, 2015

DOI: $10.3892 / \mathrm{ol} .2015 .3628$

\begin{abstract}
The level of Epstein-Barr virus DNA (EBV-DNA) in the plasma prior and subsequent to treatment is a reliable biomarker for the screening, diagnosis, monitoring and prognosis of nasopharyngeal carcinoma (NPC). The present retrospective study aimed to determine whether pre- and post-treatment levels of plasma EBV-DNA were predictive of survival in a large sample of patients with NPC. The level of plasma EBV-DNA in 637 NPC patients prior and subsequent to treatment was determined by quantitative polymerase chain reaction. The value of pre- and post-treatment plasma EBV-DNA in predicting the survival of NPC patients was then analyzed. The results revealed that pre-treatment plasma EBV-DNA loads were significantly higher in patients with NPC than those in healthy controls $(\mathrm{P}<0.001)$. The percentage of patients with positive plasma EBV-DNA was markedly higher prior to treatment $(70.64 \%$; median, 1150 copies $/ \mathrm{ml}$; range, $0-9.75 \times 10^{6}$ copies $\left./ \mathrm{ml}\right)$ than following treatment $(25.99 \%$; median, 0 copies $/ \mathrm{ml}$; range, $0-3.83 \times 10^{6}$ copies $\left./ \mathrm{ml}\right)(\mathrm{P}<0.001)$. Patients with a high plasma EBV-DNA load presented with a higher clinical tumor classification, lymph node status, metastatic status and overall cancer stage. The risk of NPC relapse and mortality was higher in patients with pre-treatment plasma EBV-DNA levels of $\geq 1,500$ copies $/ \mathrm{ml}$ than that in patients with $<1,500$ copies $/ \mathrm{ml}$. Furthermore, the risk of relapse and mortality was higher in patients with positive post-treatment plasma EBV-DNA than in patients with negative post-treatment plasma EBV-DNA. Detectable post-treatment plasma EBV-DNA was
\end{abstract}

Correspondence to: Dr Xiang-Ping Li, Department of Otolaryngology - Head and Neck Surgery, Nanfang Hospital, Southern Medical University, 1838 North Guangzhou Avenue, Guangzhou, Guangdong 510515, P.R. China

E-mail: 1i321162@qq.com

${ }^{*}$ Contributed equally

Key words: nasopharyngeal carcinoma, Epstein-Barr virus DNA, prognosis the most significant prognostic factor to affect relapse-free survival, whilst metastasis was the prognostic factor with the greatest effect on overall survival. These data indicated that pre- and post-treatment levels of plasma EBV-DNA were able to predict the prognosis of NPC. This finding may provide novel references for research and clinical practice.

\section{Introduction}

Nasopharyngeal carcinoma (NPC) is a tumor of the head and neck with a complex etiology. The incidence of NPC is low in Western countries ( 0.5 per 100,000); however, in Southern China and Asia NPC incidence peaks at 30 per 100,000 (1). Although radiotherapy (2) and chemoradiotherapy (3) have significantly improved the survival rates of patients with NPC, the five-year survival probability remains low due to disease recurrence and distant metastasis (4). As a result of the high incidence of recurrence and frequently fatal metastasis, the identification of risk factors for recurrence and metastasis will benefit patients with NPC.

Previous studies have demonstrated an association between Epstein-Barr virus (EBV) infection and NPC. EBV-DNA may be detected in NPC cells $(5,6)$, and cell-free EBV-DNA may be detected in the plasma of patients with NPC (7-13). Furthermore, the levels of plasma EBV-DNA in recently diagnosed NPC patients have been significantly correlated with tumor volume $(14,15)$, response to treatment $(16)$, tumor clearance $(17,18)$ and tumor recurrence (19-21). Similarly, it has been observed that a positive post-treatment detection of plasma EBV-DNA is predictive of poor outcomes in terms of subsequent relapse rate, overall survival (OS) and relapse-free survival (RFS) $(11,13)$. Therefore, the pre- and post-treatment levels of plasma EBV-DNA have been used as reliable biomarkers for the screening, diagnosis, monitoring and prognosis of NPC. However, previous studies have included small cohorts of patients with NPC or short follow-up times.

The identification of novel risk factors involved in disease recurrence and poor survival is important for facilitating early intervention. Furthermore, these risk factors will be of significance for the prognosis and management of patients with NPC. The objective of the present retrospective study 
was to determine whether pre- and post-treatment levels of plasma EBV-DNA were predictive of poor survival in a large sample of patients with NPC.

\section{Materials and methods}

Patients and clinical specimens. In order to determine the potential value of pre- and post-treatment plasma EBV-DNA levels for the prognosis of NPC, 637 patients with newly diagnosed NPC from the Department of Otolaryngology - Head and Neck Surgery, Nanfang Hospital (Guangzhou, China) and 245 healthy controls from the Physical Examination Center, Nanfang Hospital were recruited between January 2006 and April 2013. NPC patients were diagnosed following the pathological examination of biopsied tissues, and classified according to the 2009 American Joint Committee on Cancer tumor-node-metastasis (TNM) staging system (7th edition) (22). Patients with other types of tumor were excluded from the study.

Venous blood samples were collected from patients prior to treatment and at the first follow-up appointment. In total, $8 \mathrm{ml}$ whole blood was drawn into EDTA-containing tubes (BD Biosciences, Franklin Lakes, NJ, USA) and separated into plasma and cellular fractions by centrifugation at $1,500 \mathrm{x} \mathrm{g}$ for $5 \mathrm{~min}$ at $4^{\circ} \mathrm{C}$. The plasma was then stored at $-80^{\circ} \mathrm{C}$ until further processing. The present study was approved by the ethics committee of Nanfang Hospital, Southern Medical University (Guangzhou, Guangdong, China) and written informed consent was obtained from all patients prior to treatment.

Clinical management. All patients received a uniform protocol of conventional two-dimensional radiotherapy to the primary tumor and neck region, with a total dose of 66-70 Gy over a six- to eight-week period. In addition, 516 of the $637(81.00 \%)$ patients with advanced disease (T3-T4 or N2-N3) received induction chemotherapy prior to radiation or adjuvant chemotherapy subsequent to radiation.

Following the completion of treatment, all patients were examined at 3, 6 and 12 months in the first year, every 6 months during the second and third years, and yearly thereafter. Recurrence of NPC was evaluated by clinical physical examination, chest radiography, magnetic resonance imaging and/or computed tomography (CT; from the skull base to the whole neck), abdominal sonography, whole-body bone scans, fiberoptic nasopharyngoscopy and biopsied pathological verification if necessary and if the patient agreed. Positron emission tomography-CT was used in order to confirm the presence of distant metastasis.

Quantification of plasma EBV-DNA. The detection of plasma EBV-DNA was performed by the Department of Laboratory Medicine, Nanfang Hospital. The total plasma DNA was extracted using a QIAamp Blood Kit (Qiagen, Hilden, Germany) and the content of plasma EBV-DNA was determined by quantitative polymerase chain reaction (qPCR) using an EBV qPCR kit (Liferiver, Shanghai, China), according to the manufacturer's instructions. GAPDH (house keeping gene) was used as the internal control. All reactions were performed in triplicate, including a nontemplate control
Table I. Patients and disease characteristics $(n=637)$.

\begin{tabular}{lcc}
\hline Characteristic & Value & $\mathrm{n}(\%)$ \\
\hline $\begin{array}{l}\text { Age, years } \\
\text { Median } \\
\text { Range }\end{array}$ & 46 & \\
Gender & $14-80$ & \\
Male & & \\
Female & - & $477(74.88)$ \\
Overall stage & - & $160(25.12)$ \\
I & & \\
II & - & $21(3.30)$ \\
III & - & $100(15.70)$ \\
IV & - & $244(38.30)$ \\
\end{tabular}

and a total of $5 \mu \mathrm{l}$ DNA was used for each reaction. Briefly, the following primers were used: Forward, 5'-GCTGCGCTG CTGCTATCTT-3' and reverse, 5'-CAAGCCCACTCCCCT GTCT-3' for the BamHI-W region of the EBV genome; and forward, 5'-GGCGACGCAAAAGAAGATG-3' and reverse, 5'-CCGTTGACTCCGACCTTCAC-3' for GAPDH (Life Technologies, Grand Island, NY, USA). PCR was performed under the following conditions: Initial denaturation step at $95^{\circ} \mathrm{C}$ for $10 \mathrm{~min}$, followed by 40 cycles of $95^{\circ} \mathrm{C}$ for $15 \mathrm{sec}$ and $56^{\circ} \mathrm{C}$ for $1 \mathrm{~min}$. EBV DNA copy number was calculated using the mean values for the duplicate samples, according to the following formula: $C=\mathrm{Q} \times(\mathrm{VDNA} / \mathrm{VPCR}) \times(1 / \mathrm{Vext})$. $C$ represents the target concentration in plasma (copies $/ \mathrm{ml}$ ), $\mathrm{Q}$ represents the target quantity (copies) determined by the sequence detector, VDNA represents the total volume of DNA obtained after extraction, VPCR represents the volume of DNA solution used for PCR amplification, and Vext represents the volume of plasma extracted. In the present study, 0 copies/ml was recorded if the plasma EBV-DNA was undetectable by qPCR; a positive plasma EBV-DNA load was defined as $>0$ copies $/ \mathrm{ml}$.

Statistical analyses. Previously reported cut-off values for pre- and post-treatment plasma EBV-DNA levels were used in the present study (1,500 copies/ml pre-treatment; 0 copies/ml post-treatment) $(11,13)$. Patients were stratified at $1,500 \mathrm{copies} / \mathrm{ml}$ pre-treatment and 0 copies $/ \mathrm{ml}$ post-treatment. The relapse and mortality rates of each group of patients were analyzed by the $\chi^{2}$ test. The RFS (or OS) was calculated from the first day of induction chemotherapy to the date of disease recurrence, mortality or the final follow-up visit. The periods of RFS and OS among the various groups of patients were evaluated by the Kaplan-Meier method and analyzed by the log-rank test. The potential risk of age, gender, tumor classification, lymph node status, metastasis status, and status of pre-treatment and post-treatment plasma EBV-DNA on the survival of NPC patients was analyzed by the hazard ratio (HR), 95\% confidence interval (CI) and Wald test using the multivariate Cox proportional hazards model. Levels of pre-treatment EBV-DNA between different tumor classification, lymph node status, metastasis status and overall stage 
Table II. Pre-treatment plasma EBV-DNA levels and clinical characteristics ( $\mathrm{n}=637)$.

\begin{tabular}{|c|c|c|c|c|}
\hline \multirow[b]{2}{*}{ Characteristics } & \multirow[b]{2}{*}{$\mathrm{n}(\%)$} & \multicolumn{2}{|c|}{ EBV-DNA (copies/ml) } & \multirow[b]{2}{*}{ P-value } \\
\hline & & Median & Mean rank score & \\
\hline Tumor classification & & & & ${ }^{\mathrm{a}} \mathrm{P}<0.001$ \\
\hline $\mathrm{T} 1$ & $108(16.95 \%)$ & 630 & 251.88 & \\
\hline $\mathrm{T} 2$ & $151(23.71 \%)$ & 942 & 284.63 & \\
\hline $\mathrm{T} 3$ & $191(29.98 \%)$ & 2250 & 341.05 & \\
\hline $\mathrm{T} 4$ & $187(29.36 \%)$ & 3500 & 363.00 & \\
\hline Lymph node status & & & & ${ }^{\mathrm{a}} \mathrm{P}<0.001$ \\
\hline N0 & $74(11.62 \%)$ & 0 & 205.24 & \\
\hline N1 & $191(29.98 \%)$ & 832 & 269.27 & \\
\hline $\mathrm{N} 2$ & $270(42.39 \%)$ & 2270 & 337.58 & \\
\hline N3 & $102(16.01 \%)$ & 8900 & 445.48 & \\
\hline Metastasis status & & & & ${ }^{\mathrm{b}} \mathrm{P}<0.001$ \\
\hline M0 & $606(95.13 \%)$ & 1000 & 308.71 & \\
\hline M1 & $31(4.87 \%)$ & 12200 & 520.06 & \\
\hline Overall stage & & & & ${ }^{\mathrm{a}} \mathrm{P}<0.001$ \\
\hline I & $21(3.30 \%)$ & 0 & 148.69 & \\
\hline II & $100(15.70 \%)$ & 420 & 212.58 & \\
\hline III & $244(38.30 \%)$ & 982 & 300.21 & \\
\hline IV & $272(42.70 \%)$ & 4620 & 388.13 & \\
\hline
\end{tabular}

${ }^{\mathrm{a}} \mathrm{P}$-values were determined using the Kruskal-Wallis test. ${ }^{\mathrm{b}} \mathrm{P}$-values were determined using the Wilcoxon test. EBV, Esptein-Barr virus; $\mathrm{T}$, tumor; $\mathrm{N}$, nodal; $\mathrm{M}$, metastasis.

were compared using Kruskal-Wallis and Wilcoxon tests. All statistical analyses were performed using SPSS version 19.0 for Windows (SPSS, Inc., Chicago, IL, USA). A two-tailed P-value of $<0.05$ was considered to indicate a statistically significant difference.

\section{Results}

Patient characteristics. The demographic and clinical characteristics of the 637 patients included in the present study are shown in Table I. During the median observation period of 23 months (range, 5-75 months), there were 140 patients with NPC recurrence and 101 patients who had succumbed to the disease.

Alterations in pre- and post-treatment plasma EBV-DNA. The analysis of plasma EBV-DNA was performed in 245 healthy controls, 637 NPC patients prior to treatment and 277 NPC patients subsequent to treatment. In the present study, plasma EBV-DNA was positive in $8(3.27 \%)$ healthy controls and $450(70.64 \%)$ NPC patients prior to treatment (Fig. 1A; $\mathrm{P}<0.001)$. The pre-treatment plasma EBV-DNA loads were significantly higher in NPC patients than those in healthy controls (Fig. 1B; $\mathrm{P}<0.001$ ). The percentage of NPC patients positive for EBV-DNA was significantly higher prior to treatment (70.64\%) than following treatment (25.99\%; Fig. 1C; $\mathrm{P}<0.001)$. Furthermore, the pre-treatment plasma EBV-DNA loads were significantly higher (median, 1150 copies $/ \mathrm{ml}$; range, $0-9.75 \times 10^{6}$ copies $/ \mathrm{ml}$ ) than those post-treatment (median,
0 copies/ml; range, $0-3.83 \times 10^{6}$ copies $/ \mathrm{ml}$ ) (Fig. $1 \mathrm{D} ; \mathrm{P}<0.001$ ). Of the 277 patients who were examined following treatment (Fig. 1E), 208 had been positive for plasma EBV-DNA prior to treatment. Of these 208 patients, 139 (66.83\%) were negative for plasma EBV-DNA following treatment (Fig. 1F).

Next, the associations between pre-treatment plasma EBV-DNA and patient clinical characteristics were evaluated. A significant difference in the levels of pre-treatment plasma EBV-DNA was identified with respect to tumor classification, lymph node status, metastasis status and overall stage (Table II; $\mathrm{P}<0.001)$. It was revealed that patients with a high plasma EBV-DNA load had a higher clinical tumor classification, lymph node status, metastasis status and overall cancer stage.

High pre-treatment plasma EBV-DNA levels are associated with poor prognosis. The 637 patients were divided into two groups based on a plasma EBV-DNA cut-off value of 1,500 copies/ml. Results of the stratification analyses indicated that there was a significant difference in the rates of NPC relapse and mortality between the two groups of patients $(\mathrm{P}<0.001$; Fig. $2 \mathrm{~A}$ and $\mathrm{B})$. In addition, a significant difference was identified in the length of RFS and OS between the two groups ( $\mathrm{P}<0.001$; Fig. $2 \mathrm{C}$ and $\mathrm{D})$. Accordingly, the risk of NPC relapse and mortality in patients with pre-treatment plasma EBV-DNA levels of $\geq 1,500$ copies/ml was higher than that of those with pre-treatment plasma EBV-DNA levels of $<1,500$ copies/ml.

Positive post-treatment plasma EBV-DNA is associated with poor prognosis. The 277 patients who were tested for the levels 
Table III. RFS and OS analyses using a multivariate Cox proportional hazards model.

\begin{tabular}{|c|c|c|c|c|}
\hline \multirow[b]{2}{*}{ Parameter } & \multicolumn{2}{|l|}{ RFS } & \multicolumn{2}{|l|}{ OS } \\
\hline & $\mathrm{HR}(95 \% \mathrm{CI})$ & ${ }^{\mathrm{a} P}$ & $\mathrm{HR}(95 \% \mathrm{CI})$ & ${ }^{\mathrm{a} P}$ \\
\hline Age, $\geq 45$ years vs. $<45$ years & $1.346(0.695,2.604)$ & 0.378 & $0.625(0.283,1.363)$ & 0.238 \\
\hline Gender, male vs. female & $1.124(0.720,1.753)$ & 0.607 & $1.328(0.715,2.468)$ & 0.369 \\
\hline Tumor classification, $\mathrm{T} 1, \mathrm{~T} 2, \mathrm{~T} 3, \mathrm{~T} 4$ & $1.148(0.913,1.444)$ & 0.238 & $1.322(0.939,1.860)$ & 0.110 \\
\hline Lymph node status, N0, N1, N2, N3 & $1.261(0.965,1.648)$ & 0.090 & $1.549(1.054,2.277)$ & 0.026 \\
\hline Metastasis status, M1 vs. M0 & $0.139(0.019,1.027)$ & 0.053 & $4.216(1.703,10.435)$ & 0.002 \\
\hline Pre-treatment EBV-DNA, $\geq 1500$ vs. $<1500$ copies/ml & $1.954(1.080,3.534)$ & 0.027 & $1.827(0.787,4.237)$ & 0.161 \\
\hline Post-treatment EBV-DNA, positive vs. negative & $2.306(1.427,3.727)$ & 0.001 & $1.432(0.708,2.894)$ & 0.318 \\
\hline
\end{tabular}

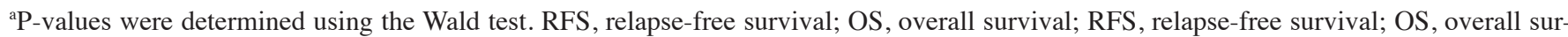
vival; T, tumor; N, nodal; M, metastasis; EBV, Esptein-Barr virus.

A

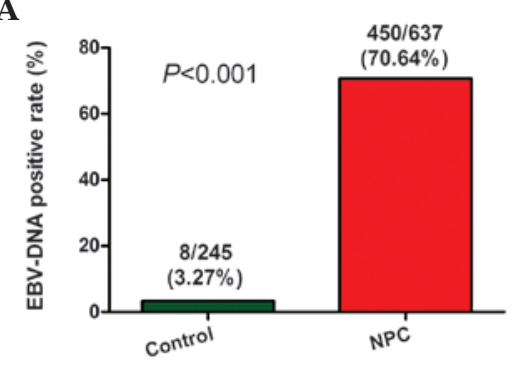

C

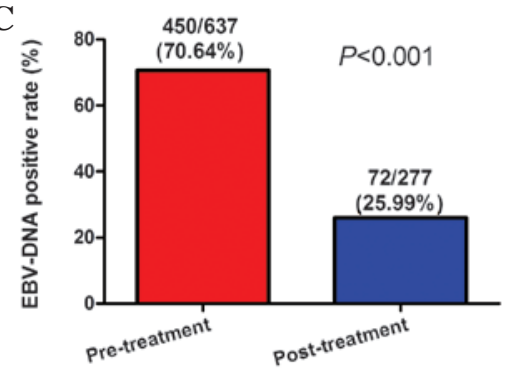

$\mathbf{E}$

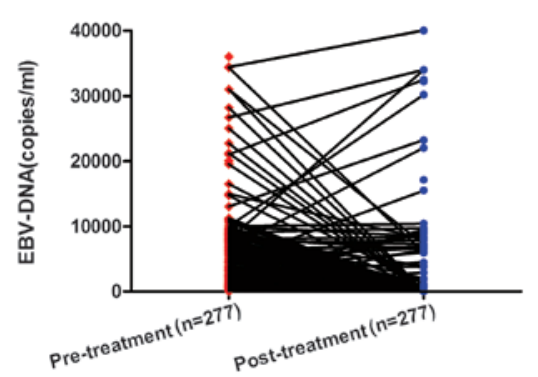

B

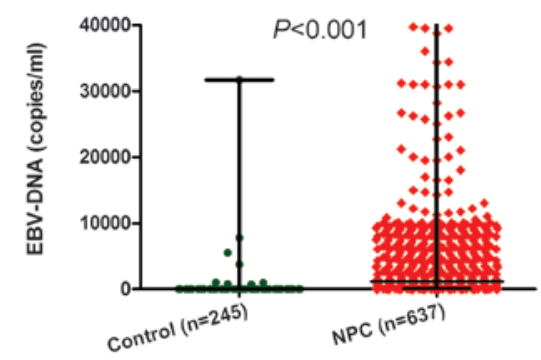

D

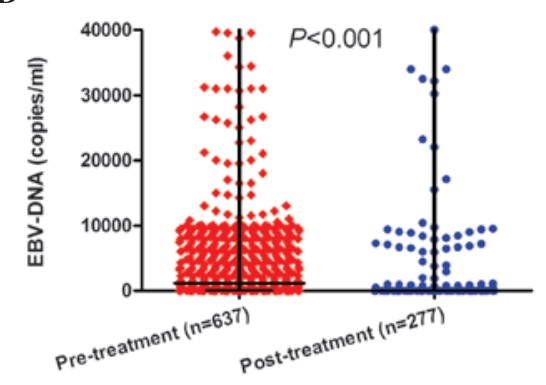

F

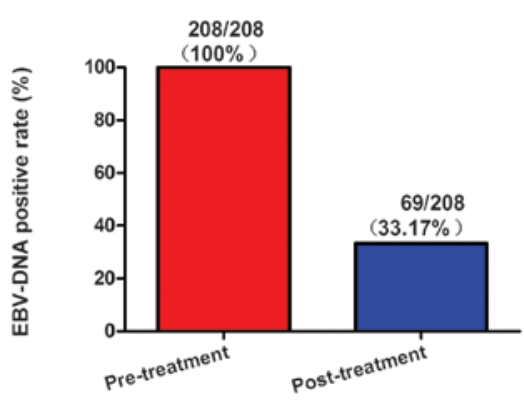

Figure 1. Changes in plasma EBV-DNA prior and subsequent to treatment. (A) Rates of positive plasma EBV-DNA detection in healthy controls and NPC patients. (B) Levels of plasma EBV-DNA in healthy controls and NPC patients. (C) Rates of positive and negative plasma EBV-DNA detection in NPC patients prior and subsequent to treatment. (D) Levels of plasma EBV-DNA in NPC patients prior and subsequent to treatment. (E) Changes in plasma EBV-DNA levels in 277 NPC patients prior and subsequent to treatment. (F) Rates of positive plasma EBV-DNA detection in 208 NPC patients prior and subsequent to treatment. EBV, Epstein-Barr virus; NPC, nasopharyngeal carcinoma.

of post-treatment plasma EBV-DNA were divided in two groups based on a plasma EBV-DNA cut-off value of 0 copies $/ \mathrm{ml}$. The relapse and mortality rates, as well as the periods of RFS and OS between the two groups of patients were then analyzed. A significant difference in the relapse and mortality rates was identified between the two groups $(\mathrm{P}<0.001$; Fig. $3 \mathrm{~A}$ and $\mathrm{B})$. Similarly, there was a significant difference in the length of RFS ( $\mathrm{P}<0.001$; Fig. 3C) and OS ( $\mathrm{P}=0.002$; Fig. 3D) between the 
A
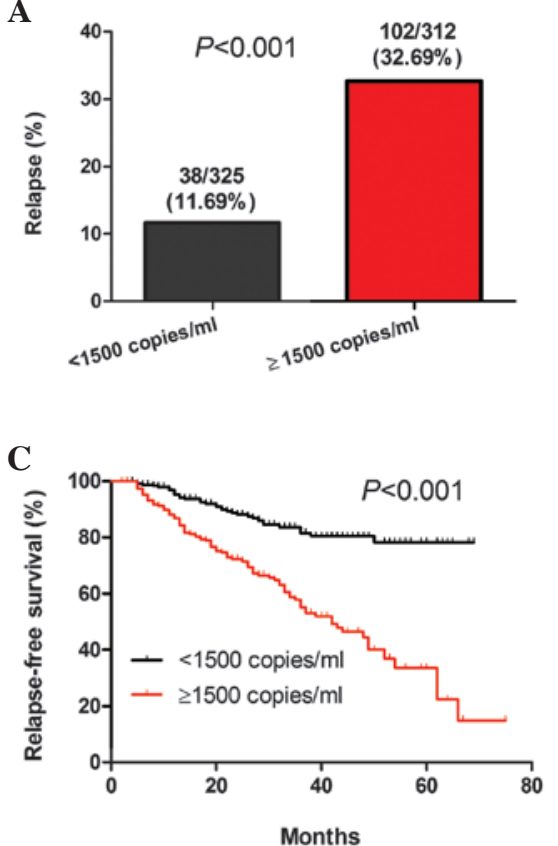

B

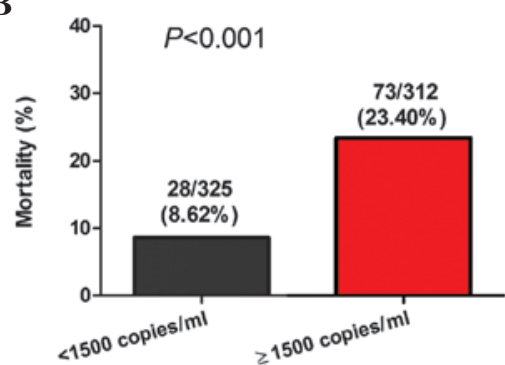

D

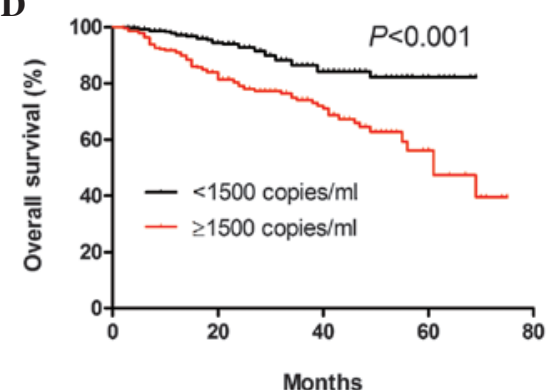

Figure 2. High pre-treatment levels of EBV-DNA are associated with increased risk or relapse and mortality. Stratification analyses of the survival of NPC patients. All NPC patients were stratified according to pre-treatment plasma EBV-DNA levels. The NPC relapse and mortality rates were then calculated. Subsequently, the RFS and OS periods of individual groups of patients were estimated by the Kaplan-Meier method and analyzed by the log-rank test. (A) Percentages of patients with relapse, (B) mortality, (C) RFS and (D) OS throughout the observation period in the two patient groups. EBV, Epstein-Barr virus; NPC, nasopharyngeal carcinoma; RFS, relapse-free survival; OS, overall survival.

A

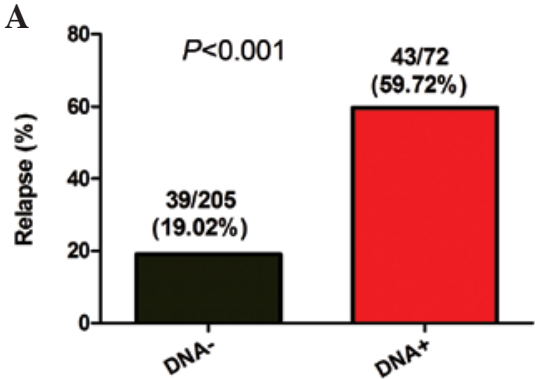

C

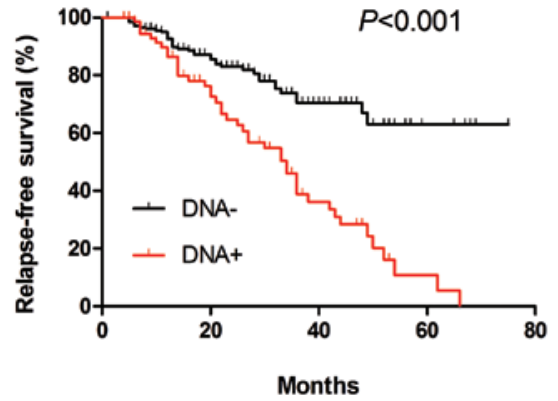

B

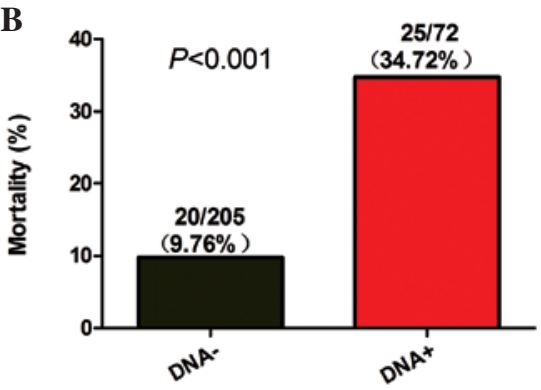

D

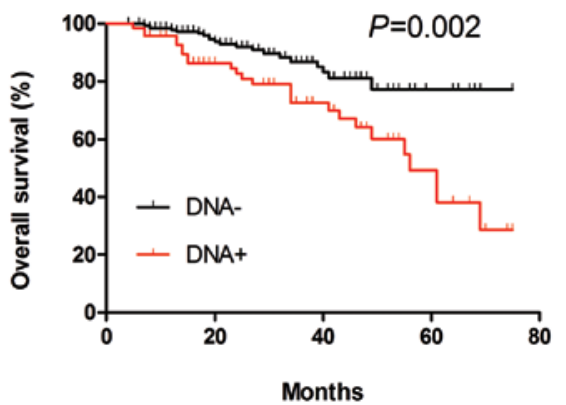

Figure 3. Detection of post-treatment EBV-DNA in NPC patients indicates a poor prognosis. Stratification analyses of the survival of NPC patients. All NPC patients were stratified according to the detection of post-treatment plasma EBV-DNA. The NPC relapse and mortality rates were then calculated. Subsequently, the RFS and OS periods of individual groups of patients were estimated by the Kaplan-Meier method and analyzed by the log-rank test. (A) Percentages of relapse, (B) mortality, (C) RFS and (D) OS throughout the observation period in the two groups of patients. EBV, Epstein-Barr virus; NPC, nasopharyngeal carcinoma; RFS, relapse-free survival; OS, overall survival.

two groups of patients. These data indicated that the risk for relapse and mortality was higher amongst NPC patients with positive post-treatment plasma EBV-DNA than that in patients with negative post-treatment plasma EBV-DNA.
Positive post-treatment EBV-DNA and the presence of distant metastasis are significant prognostic factors for RFS and OS. Multivariate analysis, using the multivariate Cox proportional hazard model, was applied to the data of the 277 patients who 
were tested for post-treatment plasma EBV-DNA. The Cox analysis was based on age, gender, tumor classification, lymph node status, metastasis, pre-treatment plasma EBV-DNA levels and post-treatment detection of plasma EBV-DNA. Risk factors that conferred a significantly shorter RFS period in patients with NPC were high pre-treatment levels of plasma EBV-DNA ( $\geq 1,500$ copies/ml; HR, 1.954; 95\% CI, 1.080-3.534; $\mathrm{P}=0.027)$ and a detectable post-treatment level of plasma EBV-DNA (HR, 2.306; 95\% CI, 1.427-3.727; $\mathrm{P}=0.001$ ) (Table III). Significant risk factors for a shorter OS period in patients with NPC, included greater lymph node involvement (HR, 1.549; 95\% CI, 1.054-2.277; P=0.026) and distant metastasis (HR, 4.216; 95\% CI, 1.703-10.435; $\mathrm{P}=0.002$ ). A detectable level of post-treatment plasma EBV-DNA was the most important prognostic factor for RFS, whilst the presence of distant metastasis was identified to be the most significant prognostic factor for OS.

\section{Discussion}

Elevated levels of circulating cell-free EBV-DNA are able to be detected by qPCR in the plasma and serum of patients with NPC (7-13). Although specificity is approximately identical in the two sample types, it has been established that the detection of EBV-DNA is more sensitive in plasma than in serum (23). Plasma EBV-DNA has become a well-known prognostic factor for the relapse and survival of NPC patients $(11-13,19)$. Similarly, the presence of serum immunoglobulin (Ig)A antibodies against the EBV capsid antigen (VCA-IgA) has also been identified to be associated with an increased risk of NPC $(24,25)$. It has been demonstrated that NPC patients with higher levels of VCA-IgA have poorer prognoses $(26,27)$. Given that plasma EBV-DNA is superior to VCA-IgA in sensitivity, specificity and predicting the prognosis of patients with NPC (28), plasma EBV-DNA may replace the conventional VCA-IgA antibody test in the diagnosis and management of NPC.

Previous studies have revealed that EBV-DNA loads in NPC patients were increased as the cancer stage increased from stage I to IV (29-31). In the present retrospective study, the associations between pre-treatment levels of plasma EBV-DNA and the clinical characteristics of 637 NPC patients were investigated. It was identified that pre-treatment levels of plasma EBV-DNA in patients with NPC correlated with clinical staging, tumor classification and lymph node status, as well as with metastasis status. The most significant difference in the pre-treatment levels of plasma EBV-DNA was between varying metastasis statuses (1,000 vs. 12,200 copies $/ \mathrm{ml})$. The results also revealed that the plasma EBV-DNA loads and the rates of positive detection in NPC patients declined significantly following treatment. These data suggested that the pre-treatment levels of plasma EBV-DNA reflect tumor burden, which is consistent with the hypothesis that plasma EBV-DNA is derived from tumor cells in patients with NPC (7).

Patients with NPC are routinely tested for EBV-DNA, as it has been established that high levels of pre-treatment plasma EBV-DNA or positive post-treatment plasma EBV-DNA are prognostic factors for the relapse and survival of NPC patients. Lin et al (11) and Wang et al (13) reported that patients with pre-treatment plasma EBV-DNA levels of $>1,500$ copies $/ \mathrm{ml}$ or persistently detectable post-treatment plasma EBV-DNA had a higher probability of treatment failure. Lin et al (11) also identified that pre-treatment plasma EBV-DNA levels were lower in patients with local recurrence than in those with distant metastasis (1,311 vs. 4,253 copies/ml) (11). A further study by Leung et al (19) found that high pre-treatment plasma EBV-DNA loads in early-stage NPC ( $\geq 4,000$ copies/ml) was predictive of distant metastasis (19). In the present study, it was revealed that patients with pre-treatment plasma EBV-DNA levels of $\geq 1,500$ copies/ml exhibited poorer RFS and OS than patients with pre-treatment plasma EBV-DNA levels of $<1,500$ copies/ml. Furthermore, patients with positive post-treatment plasma EBV-DNA had significantly shorter survival periods than those who tested negative. The multivariate analyses established that pre-treatment plasma EBV-DNA levels of $\geq 1,500$ copies $/ \mathrm{ml}$ and positive post-treatment plasma EBV-DNA were risk factors associated with the recurrence of NPC. With respect to predicting mortality, however, lymph node metastasis and distant metastasis status were superior to pre- or post-treatment levels of plasma EBV-DNA.

To the best of our knowledge, the sample size of the present study was larger than that of any previous study investigating the use of plasma EBV-DNA for predicting the prognosis of patients with NPC. The findings of the present study may therefore provide novel reference points for research and clinical practice. However, certain limitations existed, including the fact that it was a retrospective study with a limited sample size and follow-up period. Therefore, the results of the present study require validation from a prospective study with a larger population and longer follow-up time.

In conclusion, the data indicated that the levels of plasma EBV-DNA prior and subsequent to treatment were predictive of the prognosis of patients with NPC. The analysis of pre- and post-treatment plasma EBV-DNA may be valuable for elucidating the prognosis of NPC in a clinical setting. Therefore, the findings of the present study may provide a novel reference point for the research and management of patients with NPC.

\section{Acknowledgements}

This study was supported by grants from the National Science Foundation of China - Guangdong Joint Fund (no. U1132003), the Natural Science Foundation of Guangdong Province (no. S2013010015685) and the Foundation for Distinguished Young Talents in Higher Education of Guangdong, China (no. 2012LYM_0039).

\section{References}

1. Wei WI and Sham JS: Nasopharyngeal carcinoma. Lancet 365: 2041-2054, 2005.

2. Lee N, Xia P, Quivey JM, et al: Intensity-modulated radiotherapy in the treatment of nasopharyngeal carcinoma: An update of the UCSF experience. Int J Radiat Oncol Biol Phys 53: 12-22, 2002.

3. Lin JC, Jan JS, Hsu CY, Liang WM, Jiang RS and Wang WY: Phase III study of concurrent chemoradiotherapy versus radiotherapy alone for advanced nasopharyngeal carcinoma: Positive effect on overall and progression-free survival. J Clin Oncol 21: 631-637, 2003.

4. Lee AW, Sze WM, Au JS, et al: Treatment results for nasopharyngeal carcinoma in the modern era: The Hong Kong experience. Int J Radiat Oncol Biol Phys 61: 1107-1116, 2005. 
5. Chang YS, Tyan YS, Liu ST, Tsai MS and Pao CC: Detection of Epstein-Barr virus DNA sequences in nasopharyngeal carcinoma cells by enzymatic DNA amplification. J Clin Microbiol 28 2398-2402, 1990.

6. Pathmanathan R, Prasad U, Chandrika G, Sadler R, Flynn K and Raab-Traub N: Undifferentiated, nonkeratinizing and squamous cell carcinoma of the nasopharynx. Variants of Epstein-Barr virus-infected neoplasia. Am J Pathol 146: 1355-1367, 1995.

7. Lo YM, Chan LY, Lo KW, et al: Quantitative analysis of cell-free Epstein-Barr virus DNA in plasma of patients with nasopharyngeal carcinoma. Cancer Res 59: 1188-1191, 1999.

8. Chan AT, Lo YM, Zee B, et al: Plasma Epstein-Barr virus DNA and residual disease after radiotherapy for undifferentiated nasopharyngeal carcinoma. J Natl Cancer Inst 94: 1614-1619, 2002.

9. Adham M, Greijer AE, Verkuijlen SA, et al: Epstein-Barr virus DNA load in nasopharyngeal brushings and whole blood in nasopharyngeal carcinoma patients before and after treatment. Clin Cancer Res 19: 2175-2186, 2013.

10. Chan KC, Hung EC, Woo JK, et al: Early detection of nasopharyngeal carcinoma by plasma Epstein-Barr virus DNA analysis in a surveillance program. Cancer 119: 1838-1844, 2013.

11. Lin JC, Wang WY, Chen KY, et al: Quantification of plasma Epstein-Barr virus DNA in patients with advanced nasopharyngeal carcinoma. N Engl J Med 350: 2461-2470, 2004.

12. Wang WY, Twu CW, Chen HH, et al: Plasma EBV DNA clearance rate as a novel prognostic marker for metastatic/recurrent nasopharyngeal carcinoma. Clin Cancer Res 16: 1016-1024, 2010.

13. Wang WY, Twu CW, Chen $\mathrm{HH}$, et al: Long-term survival analysis of nasopharyngeal carcinoma by plasma Epstein-Barr virus DNA levels. Cancer 119: 963-970, 2013.

14. Fan H, Nicholls J, Chua D, et al: Laboratory markers of tumor burden in nasopharyngeal carcinoma: A comparison of viral load and serologic tests for Epstein-Barr virus. Int J Cancer 112 1036-1041, 2004.

15. Chan KC, Chan AT, Leung SF, et al: Investigation into the origin and tumoral mass correlation of plasma Epstein-Barr virus DNA in nasopharyngeal carcinoma. Clin Chem 51: 2192-2195, 2005.

16. Hsu CL, Chang KP, Lin CY, et al: Plasma Epstein-Barr virus DNA concentration and clearance rate as novel prognostic factors for metastatic nasopharyngeal carcinoma. Head Neck 34 1064-1070, 2012.

17. Lo YM, Leung SF, Chan LY, et al: Kinetics of plasma Epstein-Barr virus DNA during radiation therapy for nasopharyngeal carcinoma. Cancer Res 60: 2351-2355, 2000.

18. To EW, Chan KC, Leung SF, et al: Rapid clearance of plasma Epstein-Barr virus DNA after surgical treatment of nasopharyngeal carcinoma. Clin Cancer Res 9: 3254-3259, 2003.

19. Leung SF, Chan AT, Zee B, et al: Pretherapy quantitative measurement of circulating Epstein-Barr virus DNA is predictive of posttherapy distant failure in patients with early-stage nasopharyngeal carcinoma of undifferentiated type. Cancer 98: 288-291, 2003.
20. Ferrari D, Codecà $\mathrm{C}$, Bertuzzi $\mathrm{C}$, et al: Role of plasma EBV DNA levels in predicting recurrence of nasopharyngeal carcinoma in a Western population. BMC Cancer 12: 208, 2012.

21. Chan JY and Wong ST: The role of plasma Epstein-Barr virus DNA in the management of recurrent nasopharyngeal carcinoma. Laryngoscope 124: 126-130, 2014.

22. Edge SB, Byrd DR and Compton CC, et al (eds): Pharynx. In: AJCC Cancer Staging Manual. 7th edition. Springer-Verlag, New York, NY, p42, 2009.

23. Yip TT, Ngan RK, Fong AH and Law SC: Application of circulating plasma/serum EBV DNA in the clinical management of nasopharyngeal carcinoma. Oral Oncol 50: 527-538, 2014.

24. Chien YC, Chen JY, Liu MY, et al: Serologic markers of Epstein-Barr virus infection and nasopharyngeal carcinoma in Taiwanese men. N Engl J Med 345: 1877-1882, 2001.

25. Cao SM, Liu Z, Jia WH, et al: Fluctuations of Epstein-Barr virus serological antibodies and risk for nasopharyngeal carcinoma: A prospective screening study with a 20 -year follow-up. PLoS One 6: e19100, 2011.

26. Ji MF, Wang DK, Yu YL, et al: Sustained elevation of Epstein-Barr virus antibody levels preceding clinical onset of nasopharyngeal carcinoma. Br J Cancer 96: 623-630, 2007.

27. Cai YL, Li J, Lu AY, et al: Prognostic significance of serum anti-Epstein-Barr virus antibodies in nasopharyngeal carcinoma. Zhonghua Shi Yan He Lin Chuang Bing Du Xue Za Zhi 27: 119-122, 2013 (In Chinese).

28. Twu CW, Wang WY, Liang WM, et al: Comparison of the prognostic impact of serum anti-EBV antibody and plasma EBV DNA assays in nasopharyngeal carcinoma. Int J Radiat Oncol Biol Phys 67: 130-137, 2007.

29. Lo YM, Leung SF, Chan LY, et al: Plasma cell-free Epstein-Barr virus DNA quantitation in patients with nasopharyngeal carcinoma. Correlation with clinical staging. Ann NY Acad Sci 906: 99-101, 2000.

30. Leung SF, Lo YM, Chan AT, et al: Disparity of sensitivities in detection of radiation-naïve and postirradiation recurrent nasopharyngeal carcinoma of the undifferentiated type by quantitative analysis of circulating Epstein-Barr virus DNA1,2. Clin Cancer Res 9: 3431-3434, 2003.

31. Hou X, Zhao C, Guo Y, et al: Different clinical significance of pre- and post-treatment plasma Epstein-Barr virus DNA load in nasopharyngeal carcinoma treated with radiotherapy. Clin Oncol (R Coll Radiol) 23: 128-133, 2011. 